\title{
VULNERABILIDAD DE PADECER DIABETES MELLITUS TIPO 2 EN LA COMUNIDAD DE JOA
}

AUTORES: Barbara Miladys Placencia López ${ }^{1}$

Arturo Hernández Escobar ${ }^{2}$

Augusto Rafael Fienco Bacusoy ${ }^{3}$

Allison Vanessa Reyes Chele ${ }^{4}$

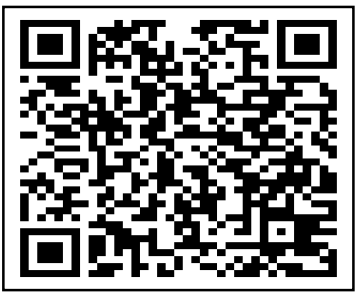

DIRECCIÓN PARA CORRESPONDENCIA: miladys.placencia@unesum.edu.ec

Fecha de recepción: 20/12/2020

Fecha de aceptación: 22/03/2021

\section{RESUMEN}

La diabetes mellitus es una enfermedad crónica no transmisible (ECNT) que tiene una alta prevalencia en el mundo, los inadecuados estilos de vida y el autocuidado de la población son factores que inciden negativamente en esta problemática. El presente estudio se desarrolló en la comunidad de Joa perteneciente al cantón Jipijapa-Manabí-Ecuador, tuvo como propósito determinar la vulnerabilidad de padecer diabetes tipo 2 en la población adulta en las edades comprendidas entre 20 y 60 años de edad. Se realizó un estudio descriptivo, transversal, y de análisis a personas con riesgo de padecer diabetes en usuarios de 20 años en adelante, se realizó una encuesta para la recogida de información, se midió, además, el índice de masa corporal (IMC) y la glucemia. La hipertensión y la diabetes son las enfermedades que más afectan a la comunidad objeto de estudio, y los riesgos de padecer diabetes se relacionaron con los valores de glucemia por encima de los valores normales, la obesidad y la falta de ejercicios físicos, alteraciones que afectaron en mayor porcentaje al género femenino, sumado a la notable afectación económica existente para el adecuado cuidado de la salud.

PALABRAS CLAVE: Prevalencia; índice de masa corporal; valores de glucemia; obesidad.

\section{VULNERABILITY OF SUFFERING FROM TYPE 2 DIABETES MELLITUS IN THE COMMUNITY OF JOA}

\author{
ABSTRACT \\ ${ }^{1}$ Docente de la Carrera de Enfermería, Facultad de la Salud, UNESUM, Km 1.5 Vía Noboa, Jipijapa 130650, \\ Jipijapa, Ecuador, E-mail: miladys.placencia@unesum.edu.ec \\ ${ }^{2}$ Docente de la Carrera Ingeniería ambiental, Facultad de Ciencias Naturales y de la Agricultura, UNESUM, Km 1.5 \\ Vía Noboa, Jipijapa 130650, Jipijapa, Ecuador, E-mail: arturo.hernandez@unesum.edu.ec, https://orcid.org/0000- \\ 0001-8403-6163 \\ ${ }^{3}$ docente de la Carrera Ingeniería ambiental, Facultad de Ciencias Naturales y de la Agricultura, UNESUM, Km 1.5 \\ Vía Noboa, Jipijapa 130650, Jipijapa, Ecuador, E-mail: augusto.fienco@unesum.edu.ec \\ ${ }^{4}$ Lic. en Enfermería graduada en la UNESUM, Km 1.5 Vía Noboa, Jipijapa 130650, Jipijapa, Ecuador, E-mail: \\ allireyes@hotmail.com
}


Diabetes mellitus is a chronic non-communicable disease (NCD) that has a high prevalence in the world, inadequate lifestyles and self-care of the population are factors that negatively affect this problem. The present study was developed in the community of Joa belonging to the JipijapaManabí-Ecuador canton, its purpose was to determine the vulnerability of suffering from type 2 diabetes in the adult population in the ages between 20 and 60 years of age. A descriptive, crosssectional and analytical study was carried out on people at risk of suffering from diabetes in users 20 years of age and older, a survey was carried out to collect information, and the body mass index (BMI) and blood glucose. Hypertension and diabetes are the diseases that most affect the community under study, and the risks of suffering from diabetes were related to blood glucose values above normal values, obesity and lack of physical exercise, alterations that affected in greater percentage to the feminine gender, added to the notable economic affectation existing for the adequate health care.

KEYWORDS: Prevalence; body mass index; blood glucose values; obesity.

\section{INTRODUCCIÓN}

La Diabetes Mellitus constituye uno de los mayores problemas del siglo XXI, principalmente en las sociedades desarrolladas, actualmente es considerada como una enfermedad degenerativa, la cual es uno de los peligros de la salud pública más importante a nivel mundial, que afecta a niños, adolescentes y a personas en la edad adulta, por distintos factores de riesgo como el sobrepeso, obesidad, aumento de glucosa en sangre provocada por una deficiencia insulina producida por el páncreas, son factores desencadenantes para el desarrollo de la enfermedad (OMS, 2020)

Se conoce mundialmente según las estimaciones, que 422 millones de adultos fueron diagnosticados con Diabetes Mellitus en el año 2014, frente a los 108 millones en 1980, por lo que se puede notar que la comunidad de personas con esta patología ha aumentado progresivamente, se considera además que estas cifras continuarán creciendo, mientras no se adopten acciones drásticas para su control, y prevención, por lo tanto como es una enfermedad silenciosa, su marcha está siendo implacable y tenemos que detenerla, menciona Krug, responsable de la Organización Mundial de la Salud (OMS) en la lucha contra este padecimiento (Krug, 2016)

En América Latina y el Caribe la diabetes tiene alta prevalencia, la cual presenta porcentajes de $12,4 \%$ en Belice, y en México estos resultados oscilan los 10,7\%, cabe mencionar que en las ciudades de Guatemala y Bogotá esta se mantiene en un 8 a 10\%, la situación cambió de manera drástica, donde las incidencias entre las fronteras de México y Estados Unidos aumentan en un 16\% (Perez R, 2016)

Es importante considerar que la atención que brinda el sistema sanitario de salud de cada país que conforma el continente americano, se maneja de manera diferente, pero direccionadas con un mismo propósito, disminuir y controlar el crecimiento de esta enfermedad.

En Ecuador la tasa de muertos ha tenido un incremento significativo en los últimos años a causa de la Diabetes Mellitus, con un total de 4.895 fallecimientos durante el 2017, la cual podría aumentar debido a los inadecuados estilos de vida como el sedentarismo, sumándose como elementos importantes, otros factores que influyen en la esfera psicosocial, destacándose entre ellos el desempleo, los problemas económicos, la desintegración familiar, entre otros (Zavala A, 2018) 
Incremento que está relacionado con los inadecuados estilos de vida en muchos de los casos, y agudizado por los problemas económicos que atraviesa el país y el consiguiente desempleo de la población.

Según Duran (2020), de acuerdo con lo manifestado por el Ministerio de Salud Pública (MSP), en el Ecuador actualmente existe una prevalencia que equivale a un $8,5 \%$ de la población que padece esta patología. Por ello esta institución ha trazado estrategias vinculadas a la prevención, tratamiento y diagnóstico oportuno, con el propósito de estandarizar su manejo, con ello garantizar de esta manera que se brinde una atención médica de calidad, tanto en los establecimientos públicos como privados, con el fin disminuir la incidencia de esta enfermedad que cada vez aumenta progresivamente, y causa complicaciones severas en la población que la padece.

Los riesgos de padecer Diabetes son cada vez más altos en la población, a pesar de los programas de salud existentes, que incluyen actividades de promoción de salud y prevención de enfermedades, y otras acciones estratégicas dirigidas a las comunidades; medidas que aún no son suficientes para el problema sanitario existente, y que requieren de un mejor seguimiento y control por parte de las entidades de salud pública.

La diabetes mellitus es una enfermedad crónica no transmisible (ECNT) que tiene una alta prevalencia en el mundo, los inadecuados estilos de vida y el autocuidado de la población son factores que inciden negativamente en esta problemática. El presente estudio se desarrolló en la comunidad de Joa perteneciente al cantón Jipijapa-Manabí-Ecuador, tuvo como propósito determinar la vulnerabilidad de padecer diabetes tipo 2 en la población adulta en las edades comprendidas entre 20 y 60 años de edad

\section{DESARROLLO}

Se realizó un estudio descriptivo, transversal, y de análisis en la comunidad de Joa, perteneciente al cantón Jipijapa, con un total de 371 habitantes, se consideró como muestra 40 personas en riesgo de padecer Diabetes Mellitus, las técnicas que se utilizaron fue la observación, la aplicación de encuestas, donde se obtuvo información relevante de las variables de investigación, posteriormente se realizó la toma de glucemia en ayunas, la medición de peso, talla y perímetro abdominal, se consideró como criterios de inclusión a personas de 20 años en adelante, aquellas que estuvieron de acuerdo en participar en la investigación, con antecedentes familiares y personales, glicemia elevada, que tengan sobrepeso y obesidad, y entre los criterios de exclusión se consideró a personas que no tuvieron riesgo predisponente para desarrollar la enfermedad, y las que se negaron a participar en esta investigación. 
Barbara M. Placencia López, Arturo Hernández Escobar , Augusto R. Fienco Bacusoy, Allison Vanessa Reyes Chele

\section{Resultados y discusión}

Tabla 1: Antecedentes Patológicos Personales

\begin{tabular}{|c|c|c|c|c|c|c|}
\hline & & PORCE & & PORCE & TOTAL & TOTAL \\
\hline ENFERMEDAD & HOMBRE & NTAJE & MUJER & NTAJE & & \\
\hline Cáncer & - & $0 \%$ & 1 & $4 \%$ & 1 & $3 \%$ \\
\hline $\begin{array}{l}\text { Diabetes o azúcar } \\
\text { elevada en la sangre }\end{array}$ & 5 & $36 \%$ & 9 & $35 \%$ & 14 & $35 \%$ \\
\hline $\begin{array}{l}\text { Enfermedades del } \\
\text { corazón }\end{array}$ & - & $0 \%$ & 1 & $4 \%$ & 1 & $3 \%$ \\
\hline $\begin{array}{l}\text { Hipertensión o presión } \\
\text { arterial alta }\end{array}$ & 6 & $43 \%$ & 9 & $35 \%$ & 15 & $38 \%$ \\
\hline $\begin{array}{l}\begin{array}{l}\text { Nefropatía } \\
\text { enfermedad de los }\end{array} \\
\text { lo }\end{array}$ & & & & & & \\
\hline riñones & 2 & $14 \%$ & 1 & $4 \%$ & 3 & $8 \%$ \\
\hline Ninguna & 1 & $7 \%$ & 5 & $19 \%$ & 6 & $15 \%$ \\
\hline Total general & 14 & $100 \%$ & 26 & $100 \%$ & 40 & $100 \%$ \\
\hline
\end{tabular}

Nota. Fuente: Autores

$\begin{array}{lll}\text { Promedio } & 3,5 & 4,33 \\ \text { Varianza } & 5,67 & 16 \\ \text { Desviación estándar } & 2,38 & 4,00 \\ \text { Coeficiente de variación } & 68 \% & 92 \%\end{array}$

La tabla 1, demostró que la enfermedad que más afecta a los hombres es la hipertensión con un total del $43 \%$, mientras que la enfermedad que más padecen las mujeres es la diabetes junto con la hipertensión con 35\% cada una. Al total de los encuestados la enfermedad que más los afecta es la hipertensión con 38\%. El género que más coeficiente de variación demostró en el diagnóstico de enfermedades fueron las mujeres con un 92\%. Comparando los resultados obtenidos con datos de la OMS (2020), que indica que la hipertensión arterial (HTA) diagnosticada en América en personas mayores de 25 años es del 35\% observamos que los resultados son similares.

Tabla 2: Manifestaciones clínicas.

\begin{tabular}{lllllll}
\hline SINTOMAS & Hombre & Mujer & Hombre & Mujer & Total & Total \\
\hline Ansiedad por comer & 1 & 1 & $7 \%$ & $4 \%$ & 2 & $5 \%$ \\
Aumento de peso & 2 & 3 & $14 \%$ & $12 \%$ & 5 & $13 \%$ \\
Fatiga & 3 & 9 & $21 \%$ & $35 \%$ & 12 & $30 \%$ \\
Irritabilidad & 2 & 5 & $14 \%$ & $19 \%$ & 7 & $18 \%$ \\
Pérdida de peso & 2 & 2 & $14 \%$ & $8 \%$ & 4 & $10 \%$ \\
Sed excesivas & 1 & 3 & $7 \%$ & $12 \%$ & 4 & $10 \%$ \\
Visión borrosa & 3 & 3 & $21 \%$ & $12 \%$ & 6 & $15 \%$ \\
Total general & 14 & 26 & $100 \%$ & $100 \%$ & 40 & $100 \%$ \\
\hline
\end{tabular}

Nota. Fuente: Autores

242 UNESUM-Ciencias. Publicación cuatrimestral. Vol. 5, Año 2021, No. 3 (Mayo-Agosto) 


$\begin{array}{lrr}\text { Promedio } & 2,00 & 3,71 \\ \text { Varianza } & 0,67 & 6,90 \\ \begin{array}{lr}\text { Desviación estándar } \\ \text { Coeficiente }\end{array} \quad 0,82 & 2,63 \\ \begin{array}{l}\text { variación } \\ \text { de }\end{array} & \\ & 41 \% & 71 \%\end{array}$

La tabla 2, detalla que los síntomas que más presentan los hombres son la fatiga y la visión borrosa con un $21 \%$ cada uno. En las mujeres el síntoma más presente es la fatiga con un $35 \%$. En el total de los encuestados el síntoma más vigente es la fatiga con un $30 \%$. El género que más alto coeficiente de variación presentó en el diagnóstico de los síntomas fueron las mujeres con un 71\%. Según Mediavilla (2011), la diabetes se manifiesta con síntomas de fatiga, visión borrosa, poliuria, polidipsia y dolor de cabeza, pero si no se la trata a tiempo se manifiesta con síntomas de hipoventilación, debilidad, coma, dolor abdominal, náuseas y vómito, consecuencias que se corresponden con los resultados en cuanto a los síntomas de fatiga en la encuesta realizada.

Tabla 3: Índice de peso por edad

\begin{tabular}{|c|c|c|c|c|c|c|c|c|c|c|c|c|}
\hline INDICE & $\begin{array}{l}20 \\
- \\
30\end{array}$ & $\begin{array}{l}31 \\
- \\
40\end{array}$ & $\begin{array}{l}41 \\
- \\
50\end{array}$ & $\begin{array}{l}51 \\
- \\
60\end{array}$ & $\begin{array}{l}61 \\
\text { años } \\
\text { en } \\
\text { adelan } \\
\text { te }\end{array}$ & $\begin{array}{l}20- \\
30\end{array}$ & $31-40$ & $\begin{array}{l}41- \\
50\end{array}$ & $\begin{array}{l}51- \\
60\end{array}$ & $\begin{array}{l}61 \\
\text { años } \\
\text { en } \\
\text { adela } \\
\text { nte }\end{array}$ & Total & Total \\
\hline $\begin{array}{l}\text { Peso } \\
\text { normal } \\
\text { Sobrepes }\end{array}$ & 1 & 1 & - & 5 & 5 & $3 \%$ & $3 \%$ & $0 \%$ & $13 \%$ & $13 \%$ & 12 & $30 \%$ \\
\hline $\begin{array}{l}\text { o } \\
\text { Obesidad }\end{array}$ & - & 5 & 2 & 4 & 8 & $0 \%$ & $13 \%$ & $5 \%$ & $10 \%$ & $20 \%$ & 19 & $48 \%$ \\
\hline $\begin{array}{l}\text { tipo I } \\
\text { Obesidad }\end{array}$ & - & 1 & - & 1 & 5 & $0 \%$ & $3 \%$ & $0 \%$ & $3 \%$ & $13 \%$ & 7 & $18 \%$ \\
\hline $\begin{array}{l}\text { mórbida } \\
\text { Total } \\
\text { general }\end{array}$ & - & 1 & - & 10 & 19 & $0 \%$ & $3 \%$ & $0 \%$ & $0 \%$ & $48 \%$ & 40 & $\begin{array}{l}5 \% \\
100 \%\end{array}$ \\
\hline
\end{tabular}

Nota. Fuente: Autores

La tabla 3, indica que la tasa más alta de sobrepeso, de obesidad tipo I y obesidad mórbida se encuentra a la edad de 61 años en adelante con un 20\%, 13\% y 3\% respectivamente.

Según el Instituo Nacional de Estadística y Censo (2010), los adultos mayores entre 50-59 años tienen un porcentaje de $75 \%$ de sobrepeso y obesidad con un índice de masa corporal (IMC) de $\geq 25$ (2), lo que demuestra que en la zona de estudio están por debajo del promedio nacional. 
Barbara M. Placencia López, Arturo Hernández Escobar , Augusto R. Fienco Bacusoy, Allison Vanessa Reyes Chele

Tabla 4: Niveles de glucosa.

\begin{tabular}{lllllll}
\hline $\begin{array}{l}\text { Etiquetas } \\
\text { fila }\end{array}$ & Hombre & Mujer & Hombre & Mujer & Total & Total \\
\hline $\begin{array}{l}<100 \mathrm{mg} / \mathrm{dl} \\
\text { (NORMAL) }\end{array}$ & 5 & 7 & $36 \%$ & $27 \%$ & 12 & $30 \%$ \\
$\begin{array}{l}100-125 \mathrm{mg} / \mathrm{dl} \\
\text { (PRE }\end{array}$ & & & & & & \\
DIABETES) & 6 & 14 & $43 \%$ & $54 \%$ & 20 & $50 \%$ \\
$\geq 126 \mathrm{mg} / \mathrm{dl}$ & & & & & & \\
$\begin{array}{l}\text { (DIABETES) } \\
\text { Total general }\end{array}$ & 3 & 5 & $21 \%$ & $19 \%$ & 8 & $20 \%$ \\
\hline
\end{tabular}

Nota. Fuente: Autores

Promedio $\quad 4,67 \quad 8,67$

Varianza 2,33 22,33

Desviación

estándar $\quad 1,53 \quad 4,73$

Coeficiente de

variación $\quad 33 \% \quad 55 \%$

La tabla 4, detalla que el nivel de glucosa con más repercusión en los hombres fue de 100-125 $\mathrm{mg} / \mathrm{dl}$ con un $43 \%$, y en las mujeres el $54 \%$ en este mismo rango de medida, resultados que confirman la presencia de una PREDIABETES en ambos casos. En general el nivel de glucosa más común es el de 100-125 mg/dl (PREDIABETES) con un total del 50\%. En el diagnóstico de glucosa el género que presento más coeficiente de variación fueron las mujeres con un $55 \%$. En contraste a los resultados de la investigación, como lo afirma el Ministerio de Salud Pública \& Instituo de Estadística y Censo (2017), para la población ecuatoriana de 10 a 59 años, la prevalencia de diabetes, definida a partir de la glucemia $\geq 126 \mathrm{mg} / \mathrm{dl}$ es $2,7 \%, 2,6 \%$ en hombres y $2,8 \%$ en mujeres, lo que demuestra que en la zona de estudio la diabetes está por encima del promedio nacional, ya que los hombres y mujeres con nivel de $\geq 126 \mathrm{mg} / \mathrm{dl}$ es de $21 \%$ y $19 \%$ respectivamente.

Tabla 5: Ejercicios

\begin{tabular}{lllllll}
\hline Ejercicio & Hombre & Mujer & Hombre & Mujer & Total & Total \\
\hline $\begin{array}{l}1 \quad \text { a } 2 \text { veces por } \\
\text { semana }\end{array}$ & 6 & 6 & $43 \%$ & $23 \%$ & 12 & $30 \%$ \\
3 o más veces por & 5 & 1 & $36 \%$ & $4 \%$ & 6 & $15 \%$ \\
semana & 3 & 19 & $21 \%$ & $73 \%$ & 22 & $55 \%$ \\
Casi nunca & 14 & 26 & $100 \%$ & $100 \%$ & 40 & $100 \%$ \\
Total general & 14 & & & & & \\
\hline
\end{tabular}

244 UNESUM-Ciencias. Publicación cuatrimestral. Vol. 5, Año 2021, No. 3 (Mayo-Agosto) 
Nota. Fuente: Autores

$\begin{array}{lrl}\text { Promedio } & 4,67 & 8,67 \\ \text { Varianza } & 2,33 & 86,33 \\ \begin{array}{l}\text { Desviación estándar } \\ \text { Coeficiente de }\end{array} & 1,53 & 9,29 \\ \text { variación } & 33 \% & 107 \%\end{array}$

La tabla 5, detalla que la mayor frecuencia para hacer ejercicios en los hombres es de 1 a 2 veces por semana con $43 \%$, mientas que las mujeres con $73 \%$ indicaron que casi nunca hacen ejercicios. El coeficiente de variación más alto en cuanto a la frecuencia de hacer ejercicios fue para las mujeres con un 107\%. El ejercicio es un componente importante en el manejo de la diabetes, de manera que puede ser utilizado para fomentar la salud y la calidad de vida de los pacientes afectados de dicha enfermedad, sin embargo, en el estudio realizado en la comunidad de Joa, podemos observar que el 55\% de los encuestados casi nunca realizan ejercicios.

Tabla 6: Salario en dólares.

\begin{tabular}{lllllll}
\hline SALARIO & Hombre & Mujer & Hombre & Mujer & Total & Total \\
\hline $50-100$ & 7 & 2 & $50 \%$ & $8 \%$ & 9 & $23 \%$ \\
$101-200$ & 4 & - & $29 \%$ & $0 \%$ & 4 & $10 \%$ \\
$201-300$ & 1 & - & $7 \%$ & $0 \%$ & 1 & $3 \%$ \\
301 & en & & & & & \\
adelante & 2 & - & $14 \%$ & $0 \%$ & 2 & $5 \%$ \\
Ninguno & & 24 & $0 \%$ & $92 \%$ & 24 & $60 \%$ \\
Total general & 14 & 26 & $100 \%$ & $100 \%$ & 40 & $100 \%$ \\
\hline
\end{tabular}

Nota. Fuente: Autores

$\begin{array}{lll}\text { Promedio } & 3,5 & 13 \\ \begin{array}{l}\text { Varianza } \\ \begin{array}{l}\text { Desviación } \\ \text { estándar }\end{array}\end{array} & 7 & 242 \\ \begin{array}{l}\text { Coeficiente } \\ \text { variación }\end{array} & 76 \% & 15,56 \%\end{array}$

La tabla 6, nos demuestra que el salario que más perciben tanto los hombres como las mujeres es el de 50 a 100 dólares al mes con un 50\% y 8\% respectivamente. Existe un $92 \%$ de mujeres que no perciben salario alguno. La variación más alta en los rangos de los salarios está presente en las mujeres con $120 \%$ de coeficiente de variación. Los hogares ecuatorianos, según el Instituo Nacional de Estadística y Censo (2014) gastan el $24,4 \%$ del ingreso en alimentos y bebidas no alcohólicas, el $14,6 \%$ en transporte, el 9,9\% en bienes y servicios de consumo, el 7,5\% en salud. Esto significaría que del ingreso promedio mensual familiar de US\$ 893,00 se dedica a salud 
Barbara M. Placencia López, Arturo Hernández Escobar , Augusto R. Fienco Bacusoy, Allison Vanessa Reyes Chele

US\$ 67,00 mensuales, cuando en realidad el costo del tratamiento mensual para la diabetes estimado es de US\$ 371,43 por paciente.

\section{CONCLUSIONES}

La hipertensión arterial y la diabetes mellitus 2 son las enfermedades que más afectan a la población estudiada, aquejando en mayor porcentaje a las mujeres, destacándose la fatiga como síntoma clínico presente en ambos géneros.

La tasa más alta de sobrepeso, de obesidad tipo I y obesidad mórbida se encuentra a la edad de 61 años en adelante, datos que alertan los riesgos predisponentes en este grupo etario.

Se evidenció la presencia de una PREDIABETES, con índices de glucemia entre 100-125 mg/dl tanto en los hombres como en las mujeres, también se evidenció que las mujeres no realizan ejercicios físicos sistemáticos.

\section{REFERENCIAS BIBLIOGRÁFICAS}

Duran, E. (2017). Ministerio de Salud Pública. Obtenido de https://www.salud.gob.ec/con-varios-eventos-a-nivelnacional-se-fomenta-la-prevencion-de-la-diabetes/.

Instituo Nacional de Estadística y Censo. (2010). Salud, bienestar y envejecimiento. Obtenido de https:/www.ecuadorencifras.gob.ec/wp-content/descargas/Presentaciones/estadisticas_adulto_mayor.pdf

Instituo Nacional de Estadística y Censo, I. (2014). Encuesta nacional de ingresos y Gastos de los Hogares urbanos y rurales 2011-2012. Obtenido de http://www.inec.gob.ec/Enighur_Analisis_ENIGHUR\%2020112012 rev.pdf, pp. 17, 23.

Krug, $\quad$ E. (2016). Redacción BBC $\quad$ Mundo. $\quad$ Obtenido de https://www.bbc.com/mundo/noticias/2016/04/160406_salud_diabetes_oms_lb

Mediavilla, J. (2011). Complicaciones de la diabetes mellitus. Diagnóstico y tratamiento. SUMERGEN, MEDICINA FAMILIAR, 132-145.

Ministerio de Salud Pública, \& Instituo de Estadística y Censo. (2017). Encuesta Nacional de Salud y Nutrición. Obtenido de https://www.ecuadorencifras.gob.ec/documentos/webinec/Estadisticas_Sociales/ENSANUT/MSP_ENSANUT-ECU_06-10-2014.pdf

OMS. (13 de Agosto de 2020). Información general sobra la HIPERTENSIÓN en el mundo. Obtenido de https://apps.who.int/iris/bitstream/handle/10665/87679/WHO_DCO_WHD_2013.2_spa.pdf;jsessionid=4A7 FE94ACE1300773744EF221C585FA0?sequence $=1$.

Perez R, M. T. (2016). Gente Saludable. Obtenido de https://blogs.iadb.org/salud/es/.

Zavala A, F. E. (2018). Diabetes mellituss tipo 2 en el Ecuador. Revista Universitaria con proyección Cientifíca, Académico y Social, 4. 